\title{
DIFERENTES DIÂMETROS DE GOTAS E EQUIPAMENTOS PARA APLICAÇÃO DE INSETICIDA NO CONTROLE DE Pseudoplusia includens
}

\author{
JOSÉ R. G. DI OLIVEIRA ${ }^{1}$, MARCELO DA C. FERREIRA ${ }^{2}$, RODRIGO A. A. ROMÁN ${ }^{3}$
}

RESUMO: Teve-se o objetivo de avaliar o espectro e a uniformidade de gotas em função de equipamentos de pulverização, volumes de calda e dosagem de inseticida na mortalidade de Pseudoplusia includens em laboratório. O trabalho foi conduzido na UNESP de Jaboticabal, sendo realizada uma aplicação sobre as lagartas com os tratamentos: dois equipamentos (bico rotativo e bico hidráulico); dois volumes de calda (17 e $50 \mathrm{~L} \mathrm{ha}^{-1}$ para o bico rotativo, e 50 e $100 \mathrm{~L} \mathrm{ha}^{-1}$ para o bico hidráulico), e duas dosagens do inseticida endosulfan $\left(0,5\right.$ e $1,0 \mathrm{~L}$ p.c. ha $\left.{ }^{-1}\right)$, segundo delineamento inteiramente casualizado, no esquema fatorial $2 \times 2 \times 2+1$ testemunha. Avaliou-se diariamente a mortalidade das lagartas até o sexto dia após a aplicação dos tratamentos. O espectro de gotas foi avaliado em aparelho medidor de tamanho de partículas, em tempo real, que determina o diâmetro das gotas do espectro pulverizado por meio do desvio de trajetória que sofrem os raios de um feixe de laser ao atingi-las. Verifica-se que, na aplicação em laboratório, onde o produto atinge diretamente o alvo, o volume pode ser reduzido para até $17 \mathrm{~L} \mathrm{ha}^{-1}$, sem prejudicar o controle de $P$. includens; a dosagem de $0,5 \mathrm{~L} \mathrm{ha}^{-1}$ do produto comercial endosulfan (recomendada para Anticarsia gemmatalis) não controla satisfatoriamente a lagarta $P$. includens; o bico rotativo produz gotas de maior uniformidade (AR: 0,52) e com menor percentagem suscetível à deriva $(3,3 \%)$, comparada à ponta de pulverização de energia hidráulica (AR:1,34 e \% gotas $\leq 100 \mu \mathrm{m}: 15,2$ ).

PALAVRAS-CHAVE: bico rotativo, espectro de gotas, lagarta falsa-medideira.

\section{DIAMETER OF DROPLETS AND DIFFERENT EQUIPMENTS FOR THE APPLICATION OF INSECTICIDE TO CONTROL Pseudoplusia includes}

\begin{abstract}
It was aimed to evaluate the spectrum and uniformity of droplets in function of sprayers, spraying volumes and rates of chemical insecticide on the mortality of Pseudoplusia includens in laboratory. The work was carried out at UNESP, Jaboticabal, SP, Brazil. The following treatments were applied over the caterpillars: two equipments (atomizer and hydraulic nozzle); two spray volumes (17 and $50 \mathrm{~L} \mathrm{ha}^{-1}$ for the atomizer and 50 and $100 \mathrm{~L} \mathrm{ha}^{-1}$ for the hydraulic nozzle); and two dosages of the insecticide endosulfan $\left(0.5\right.$ and $\left.1.0 \mathrm{~L} \mathrm{pc} \mathrm{ha}^{-1}\right)$, in fully randomized plots, in a factorial scheme $2 \times 2 \times 2$ and 1 untreated check. The caterpillars mortality was evaluated until the $6^{\text {th }}$ day after the application of treatments. The spectrum of the droplets was evaluated in a particle size analyzer in real time determining the diameter and spectrum of droplets sprayed through the laser beam of the analyser. It was verified that the spray volume can be reduced by $17 \mathrm{~L} \mathrm{ha}^{-1}$ without losses on the $P$. includens control. The doses of $0.5 \mathrm{~L} \mathrm{pc} \mathrm{ha}^{-1}$ (recommended for Anticarsia gemmatalis) did not satisfactorily control the caterpillar soybean looper. The atomizer produces droplets of higher uniformity (SPAN: 0.52) and lower percentage of droplets susceptible to drift (3.3\%) compared to hydraulic nozzle (SPAN: 1.34 e \% droplets $\leq 100 \mu \mathrm{m}: 15.2$ ).
\end{abstract}

KEYWORDS: atomizer, droplet spectrum, soybean looper.

\footnotetext{
${ }^{1}$ Eng ${ }^{\mathrm{o}}$ Agrônomo, M.Sc., Doutorando em Agronomia (Entomologia Agrícola), FCAV/UNESP, Jaboticabal - SP, joserodolfoguimaraes@yahoo.com.br

${ }^{2}$ Eng $^{\mathrm{O}}$ Agrônomo, Prof. Dr., Departamento de Fitossanidade, FCAV/UNESP, Jaboticabal - SP.

${ }^{3}$ Eng ${ }^{0}$ Agrônomo, Mestrando em Agronomia (Entomologia Agrícola), FCAV/UNESP, Jaboticabal - SP. Bolsista CNPq.

Recebido pelo Conselho Editorial em: 27-4-2009

Aprovado pelo Conselho Editorial em: 12-12-2009

Eng. Agríc., Jaboticabal, v.30, n.1, p.92-99, jan./fev. 2010
} 


\section{INTRODUÇÃO}

De maneira geral, a espécie de lagarta desfolhadora mais importante na cultura da soja ainda é a Anticarsia gemmatalis (HUBNER, 1818) (Lepidoptera: Noctuidae). Entretanto, dentre as pragas secundárias, a lagarta falsa-medideira (Pseudoplusia includens, WALKER, 1857, Lepidoptera: Noctuidae) tem ganho importância na cultura, por causar danos constantes, ocorrer regularmente e apresentar ampla distribuição geográfica, justificando a pesquisa para o seu controle efetivo.

Entre os diversos fatores que causam o aumento populacional dessa praga, a sua permanência no terço mediano das plantas de soja protege-as do inseticida aplicado via pulverização, pois as folhas da parte superior proporcionam uma barreira (HERZOG, 1980). Dessa forma, pesquisas que avaliem e identifiquem métodos para que o inseticida atinja a região de ocorrência do inseto poderão contribuir para a efetividade do tratamento fitossanitário.

Via de regra, o controle de lagartas na cultura da soja é realizado via pulverização. Nessa modalidade, a componente mais importante são as pontas de pulverização, pois influenciam diretamente na qualidade e na segurança da aplicação, uma vez que são as responsáveis pela produção e pela distribuição das gotas (MATUO, 1990). Convencionalmente, são mais utilizadas a campo as pontas de pulverização de energia hidráulica, graças à flexibilidade que a mesma oferece em diferentes situações. Entretanto, no processo de formação de gotas por meio da energia hidráulica, gotas grandes e pequenas são produzidas ao mesmo tempo, resultando em baixa uniformidade de tamanho (TEIXEIRA, 1997).

Para aumentar a uniformidade do espectro de gotas, há disponível a tecnologia CDA (Controlled Drop Application), sendo os bicos rotativos os mais comercializados e usados a campo. Esse tipo de pulverização possui vantagens importantes, uma vez que se eliminam as gotas muito pequenas (causadoras de derivas) e as muito grandes (que se perdem por escorrimento), podendo ser selecionados tamanhos de gotas mais efetivos para determinada aplicação. Uma aplicação eficiente requer cobertura adequada da superfície-alvo com gotas de tamanho apropriado.

É provável que a distribuição uniforme de um determinado diâmetro e número adequado de gotas possibilitem o sucesso da operação, mesmo que se utilize a aplicação a volume baixo (FERREIRA, 2003). A redução de volumes de aplicação é uma tendência na agricultura, uma vez que se pretende diminuir o desperdício de água, aumentar a capacidade operacional devido ao menor número de abastecimentos e, quando em aplicação no momento correto, não deve interferir no sucesso do tratamento fitossanitário (FERREIRA et al., 1998). Nesse caso, cresce a importância de conhecer qual é a melhor combinação de densidade e diâmetro de gotas, volume e concentração de ingrediente ativo na calda, para as principais pragas, cujo controle é realizado via pulverização (FERREIRA, 2003).

Dessa forma, o presente trabalho teve o objetivo de avaliar o espectro e a uniformidade de gotas em função de equipamentos de pulverização, volumes de calda e dosagem de inseticida endosulfan na mortalidade de P. includens, em laboratório.

\section{MATERIAL E MÉTODOS}

\section{Avaliação da mortalidade das lagartas}

O trabalho foi conduzido no Departamento de Fitossanidade da UNESP, Câmpus de Jaboticabal - SP. As lagartas de P. includens utilizadas no experimento foram coletadas de um campo de soja na Fazenda de Ensino, Pesquisa e Extensão da UNESP/Jaboticabal, no dia 11-22008.

A soja onde foram coletadas aproximadamente 300 lagartas estava sendo monitorada para que as lagartas a serem utilizadas no experimento viessem da mesma condição de manejo cultural, do mesmo clima e que fossem da mesma população, para assim obter maior uniformidade entre os indivíduos. As lagartas foram coletadas em uma área de aproximadamente $900 \mathrm{~m}^{2}$ de soja, da variedade Monsoy RR 8045, sendo essa plantada em 6 de dezembro de 2007. A última aplicação de 
inseticida (endosulfan) no campo ocorreu 35 dias antes da coleta. Do total de 300 lagartas coletadas, foram classificadas 180 lagartas de aproximadamente $17 \mathrm{~mm}$ para o experimento. Foram colocadas cinco lagartas em placas de Petri para receberem gotas pulverizadas com calda inseticida. Cada placa representou uma repetição.

Utilizou-se do delineamento inteiramente casualizado, num esquema fatorial 2 (equipamentos) x 2 (volumes de calda) x 2 (dosagens de inseticida) +1 (testemunha), com quatro repetições (20 lagartas por tratamento, sendo 180 no experimento). Os pulverizadores utilizados foram: 1) manual, com motor elétrico que movimenta um bico de energia centrífuga ou bico rotativo, marca Apoiotec, e 2) pulverizador costal pressurizado com ponta de pulverização de energia hidráulica, de jato plano, modelo TT 11001. Utilizaram-se os volumes de calda de 17 e $50 \mathrm{~L} \mathrm{ha}^{-1}$, para o bico rotativo, e de 50 e $100 \mathrm{~L} \mathrm{ha}^{-1}$, para o hidráulico, respectivamente, nas dosagens de 0,5 e 1,0 L p.c. do inseticida endosulfan (Endosulfan Nortox 350 EC) por hectare.

Optou-se pelo inseticida endosulfan, pois esse apresenta menor persistência, quando comparado com outros organoclorados (KAUR et al., 1997), sendo classificado como de média persistência ambiental (PERES et al., 2004). Além de ser um produto acessível ao produtor, é eficaz contra grande número de insetos-praga e amplamente utilizado na agricultura brasileira.

Em todas as caldas de pulverização, foi adicionado adjuvante organossiliconado (Silwet L-77 Ag), na concentração de $0,05 \%$, com o objetivo de aumentar a deposição e a área coberta da superfície das plantas pelas gotas aspergidas (OZEKI, 2006), devido à diminuição da tensão superficial da calda, aumentando a área de contato da gota com o alvo.

As placas de Petri com as cinco lagartas de cada repetição foram dispostas em linha para que cada tratamento fosse aplicado simultaneamente. $\mathrm{O}$ bico rotativo apresentou rotação de $2.500 \mathrm{rpm}$. O bico hidráulico operou com pressão constante de $275,7 \mathrm{kPa}$, mantida por $\mathrm{CO}_{2}$ pressurizado. Para a verificação da cobertura da calda nos tratamentos, foram colocadas duas lâminas de papel hidrossensível ao lado das placas de Petri. A aplicação foi realizada à altura de $0,4 \mathrm{~m}$ do alvo, no dia 11 de fevereiro de 2008. No momento da aplicação, a temperatura do ar era de $33,2{ }^{\circ} \mathrm{C}$, a umidade relativa do ar de $52 \%$ e ausência de ventos.

Após a aplicação, as lagartas contaminadas foram levadas para uma sala em temperatura ambiente (a temperatura variou entre 27 e $33{ }^{\circ} \mathrm{C}$ e a umidade relativa de 37 a $65 \%$ ). A sala não foi climatizada devido ao fato da possibilidade de choque climático, uma vez que as lagartas vieram de uma condição de campo onde a temperatura estava acima de $30^{\circ} \mathrm{C}$.

Diariamente, foram trocadas as folhas de soja fornecidas para alimentação. Essas folhas não estavam contaminadas com o inseticida. As lagartas foram manipuladas com bastante cuidado para minimizar o estresse causado pelo seu manuseio. A mortalidade foi avaliada diariamente até o sexto dia após a aplicação. Os valores verificados foram transformados em percentagem de mortalidade e submetidos à análise de variância, sendo as médias comparadas pelo teste de Tukey $(\mathrm{p} \leq 5 \%)$. $\mathrm{O}$ cálculo da percentagem de eficiência no controle da lagarta, para cada tratamento, foi realizado por meio da fórmula de ABBOTT (NAKANO et al., 1981).

\section{Avaliação do espectro de gotas}

Foi avaliado o espectro de gotas produzido pelos equipamentos, em 13 de março de 2008, no Laboratório de Análises do Tamanho de Partículas (LAPAR) - UNESP, Jaboticabal - SP. Foram realizadas avaliações do diâmetro e da uniformidade das gotas por meio de aparelho medidor de tamanho de partículas em tempo real (Malvern Mastersizer S, versão 2.19). O equipamento foi ajustado para avaliar gotas de 0,5 a $900 \mu \mathrm{m}$ (lente $300 \mathrm{~mm}$ ). Nesse equipamento, uma unidade óptica determina o diâmetro das gotas do espectro pulverizado por meio do desvio de trajetória que sofrem os raios de um feixe de laser ao atingi-las. O desvio que o laser sofre depende do tamanho da partícula. Quanto menor a partícula, maior é o grau de difração que o raio de luz sofre (ETHERIDGE et al., 1999). 
A ponta hidráulica foi instalada a $40 \mathrm{~cm}$ do feixe de laser. Para manter a pressão constante, utilizou-se de ar comprimido controlado com regulador de pressão de precisão. Com a finalidade de analisar o jato aspergido pela ponta, foi utilizado um oscilador que faz com que o jato de calda atravesse transversalmente o feixe de laser durante a leitura do aparelho. O movimento da ponta entre os extremos do curso do oscilador foi calibrado para 3 segundos, sendo o tamanho de gotas medido por 1,5 segundo (metade do jato aspergido pela ponta). Cada leitura do laser é realizada com intervalo de 2 milissegundos, sendo, portanto, 750 leituras em cada jato de calda aspergido no experimento. $\mathrm{O}$ bico rotativo foi posto em funcionamento lateralmente ao feixe de laser e uma coifa foi instalada para que as gotas, ao saírem do disco, não atingissem a lente do aparelho e prejudicassem a leitura. O tempo de leitura calibrado para a repetição do jato aspergido por esse bico foi de um segundo.

As condições ambientais durante o experimento foram: temperatura do ar $23,5{ }^{\circ} \mathrm{C}$; umidade relativa do ar de $40 \%$, e ausência de ventos. Os tratamentos e o delineamento experimental utilizado foram idênticos ao teste realizado com aplicação sobre as lagartas.

Foram realizadas quatro repetições da leitura para cada tratamento, tendo como objetivo avaliar o diâmetro de gotas, tal que $10 \%$ do volume total pulverizado apresentaram gotas de diâmetro inferior a este valor $\left(\mathrm{DV}_{0,1}\right)$; diâmetro correspondente àquele em que $50 \%$ do volume total pulverizado apresentaram gotas de diâmetro inferior a esse valor $\left(\mathrm{DV}_{0,5}\right)$, diâmetro correspondente àquele em que $90 \%$ do volume total pulverizado apresentaram gotas de diâmetro inferior a esse valor $\left(\mathrm{DV}_{0,9}\right)$. A uniformidade do jato aspergido pelos bicos de pulverização foi calculada por meio da amplitude relativa do espectro de gotas.

Os valores do espectro de gotas produzidas em cada tratamento foram submetidos ao teste $\mathrm{F}$ para a análise de variância, e as médias foram comparadas pelo teste de Tukey $(\mathrm{p} \leq 5 \%)$.

\section{RESULTADOS E DISCUSSÃO}

Nas avaliações de percentagem de mortalidade de Pseudoplusia includens, em função dos bicos de pulverização (Tabela 1), verifica-se que não houve diferença significativa entre os tipos de bicos de pulverização e entre volume de aplicação. Porém, a maior dosagem de endosulfan proporcionou maior mortalidade até 5 dias após as aplicações (teste de Tukey, $\mathrm{p} \leq 5 \%$ ).

TABELA 1. Médias das percentagens de mortalidade de Pseudoplusia includens em função de bicos, de volumes de aplicação e de dosagens do inseticida endosulfan. Mortality percentage averages of Pseudoplusia includens in function of nozzles types, spraying volume and doses of endosulfan insecticide.

\begin{tabular}{|c|c|c|c|c|c|c|}
\hline \multirow{2}{*}{ Variáveis } & \multicolumn{6}{|c|}{$\%$ Mortalidade } \\
\hline & $1 \mathrm{DAA}$ & $2 \mathrm{DAA}$ & $3 \mathrm{DAA}$ & $4 \mathrm{DAA}$ & $5 \mathrm{DAA}$ & $6 \mathrm{DAA}$ \\
\hline Bico hidráulico & $51,25 \mathrm{~A}$ & $58,75 \mathrm{~A}$ & $76,25 \mathrm{~A}$ & $80,00 \mathrm{~A}$ & $85,00 \mathrm{~A}$ & $88,75 \mathrm{~A}$ \\
\hline Bico rotativo & $57,50 \mathrm{~A}$ & $68,75 \mathrm{~A}$ & $80,00 \mathrm{~A}$ & $87,50 \mathrm{~A}$ & $88,75 \mathrm{~A}$ & $91,25 \mathrm{~A}$ \\
\hline Volume menor $^{(1)}$ & $52,50 \mathrm{~A}$ & $61,25 \mathrm{~A}$ & $80,50 \mathrm{~A}$ & $83,75 \mathrm{~A}$ & $87,50 \mathrm{~A}$ & $88,75 \mathrm{~A}$ \\
\hline Volume maior $^{(2)}$ & $56,25 \mathrm{~A}$ & $66,25 \mathrm{~A}$ & $76,25 \mathrm{~A}$ & $83,75 \mathrm{~A}$ & $86,25 \mathrm{~A}$ & $91,25 \mathrm{~A}$ \\
\hline Endosulfan 1,0 $\mathrm{L} \mathrm{ha}^{-1}$ & $62,50 \mathrm{~A}$ & $72,50 \mathrm{~A}$ & $87,50 \mathrm{~A}$ & $91,25 \mathrm{~A}$ & $93,75 \mathrm{~A}$ & $95,00 \mathrm{~A}$ \\
\hline Endosulfan $0,5 \mathrm{~L} \mathrm{ha}^{-1}$ & $46,25 \mathrm{~B}$ & $55,00 \mathrm{~B}$ & $38,75 \mathrm{~B}$ & $76,25 \mathrm{~B}$ & $80,00 \mathrm{~B}$ & $85,00 \mathrm{~A}$ \\
\hline Trat. x Test. $(\mathrm{F})$ & $34,51 * *$ & $13,87 * *$ & $17,54 * *$ & $23,16^{* *}$ & $20,08 * *$ & $11,11 * *$ \\
\hline
\end{tabular}

É importante considerar que as diferenças entre os volumes de calda não prejudicaram a eficiência de controle do bico rotativo, que utilizou menores volumes de aplicação. Isso é confirmado pela percentagem de eficiência, calculada pela fórmula de ABBOTT (NAKANO et al., 
1981), em que o bico rotativo, na avaliação de 6 dias após a aplicação (6 DAA), proporcionou eficiência de controle de $P$. includens cerca de $10 \%$ maior em relação ao bico hidráulico (Figura 1).

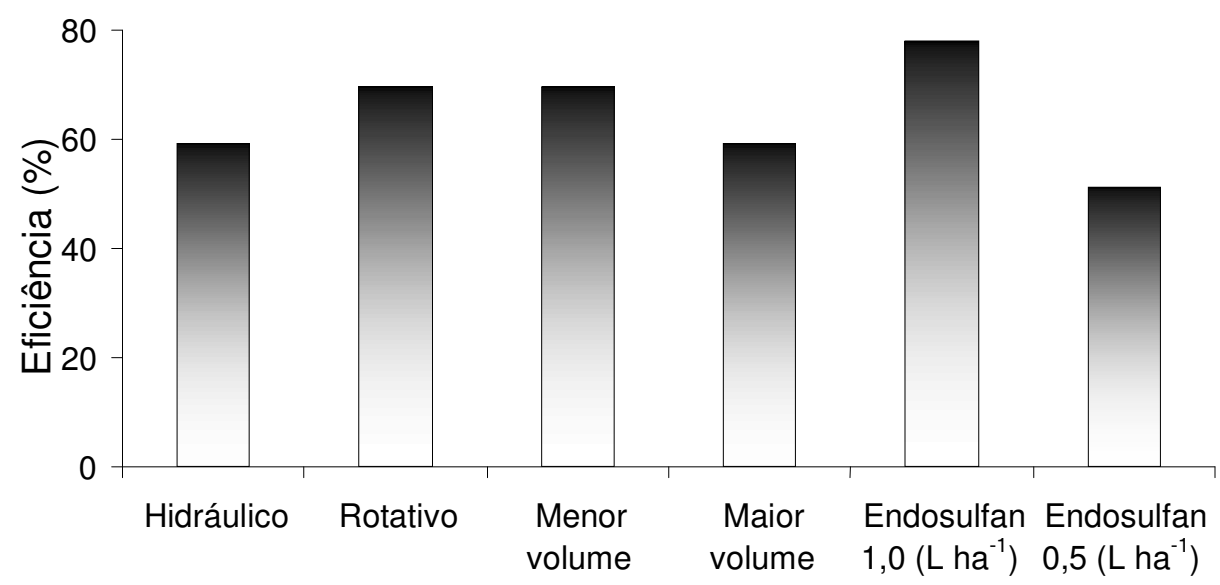

FIGURA 1. Percentagem de eficiência calculada pela fórmula de ABBOTT (NAKANO et al., 1981) do controle de Pseudoplusia includens em função dos tratamentos aplicados. (6DAA). Percentage of efficiency calculated by ABBOTT's formula (NAKANO et al., 1981) to control Pseudoplusia includens in function of the spraying treatments (6 DAA).

Isso está de acordo com COSTA et al. (2005), que afirmam que o volume de aplicação $\left(\mathrm{L} \mathrm{ha}^{-1}\right)$ não tem influência direta no resultado biológico, pois a quantidade de veículo de aplicação, por unidade de área, tem a finalidade única de diluir, transportar e facilitar a distribuição do ingrediente ativo sobre a superfície-alvo. REED \& SMITH (2001), ao avaliarem a mortalidade de Helilothis virescens por inseticidas aplicados com diferentes volumes de aplicação, verificaram que a mortalidade de lagartas tendeu a diminuir com o aumento de volume aplicado, sugerindo que a concentração de ingrediente ativo na calda seria um fator que interfere na mortalidade, pois esse fator é inversamente proporcional ao volume de aplicação. Ressalta-se que não há volume fixo de calda a ser utilizado numa área de plantio, pois esse pode variar em decorrência do alvo a ser atingido, das condições meteorológicas, dos equipamentos, do produto aplicado e do estágio de desenvolvimento da cultura.

Para a eficiência no controle, deve-se considerar a cobertura necessária em função do mínimo de volume de calda possível, uma vez que essa redução não implica perda da eficácia no tratamento. Possivelmente, a dificuldade no controle de $P$. includens no campo seja devida ao hábito de essa lagarta preferir o interior do dossel da cultura, dificultando, assim, a penetração das gotas aplicadas onde a lagarta normalmente se encontra. Para a efetividade de deposição e de penetração na aplicação de produtos fitossanitários, a qualidade e a uniformidade da cobertura do alvo estão condicionadas ao diâmetro de gotas, no qual as de menor diâmetro proporcionam maior penetração entre as folhas das culturas (SANTOS, 1992).

Com relação às dosagens do inseticida $\left(1,0 \mathrm{~L} \mathrm{ha}^{-1}\right)$, recomendadas para $P$. includens, resultou em maior mortalidade em relação à dosagem de $0,5 \mathrm{~L} \mathrm{ha}^{-1}$, indicada para A. gemmatalis (Tabela 1). No caso de que a menor dosagem houvesse produzido controle desejado, poderia contribuir para uma aplicação mais econômica no controle de duas importantes pragas na cultura da soja, pois o tratamento para controle de $A$. gemmatalis e $P$. includens seria realizado na aplicação da mesma dosagem. Entretanto, isso só será possível considerando a dosagem recomendada para a segunda espécie. PAPA \& CELOTO (2007) relatam que a espécie $P$. includens é, em geral, mais tolerante às dosagens usuais dos agrotóxicos, comparada com a A. gemmatalis. Com isso, a qualidade da aplicação requer ainda mais atenção, visando a atingir com eficiência o alvo, ou seja, as lagartas que ficam no baixeiro e no interior do dossel da cultura. 
$\mathrm{Na}$ comparação estatística, verificou-se interação entre o volume de calda e a dosagem do inseticida no quinto e sexto dias após a aplicação. O menor volume de aplicação, na dosagem de $0,5 \mathrm{~L} \mathrm{ha}^{-1}$, não controlou satisfatoriamente a lagarta $(75 \%$ de mortalidade). Porém, não houve diferença significativa de controle entre as dosagens para os volumes maiores de calda $(87,5 \mathrm{e}$ $85,0 \%$, respectivamente, para 1,0 e $0,5 \mathrm{~L}^{-1}$ e calda $\mathrm{ha}^{-1}$ ). No sexto dia, foi verificado resultado semelhante, mas a dosagem de $0,5 \mathrm{~L} \mathrm{ha}^{-1}$ proporcionou controle satisfatório $(92,5 \%)$ no maior volume de aplicação em relação ao menor volume de aplicação (77,5\%).

Isso ocorreu, possivelmente, devido à lagarta permanecer no terço médio do dossel da cultura, requerendo volumes de calda e dosagem de inseticidas cada vez maiores como forma de atingir o alvo. Isso é contrário aos fundamentos de tecnologia de aplicação de produtos fitossanitários que preconizam atingir o alvo com a quantidade necessária e com o mínimo de perdas e de contaminações do meio ambiente (MATUO, 1990).

Os tratamentos apresentaram, no geral, valores de eficiência pela fórmula de ABBOTT (NAKANO et al., 1981) entre 60 e $80 \%$ no controle de $P$. includens (Figura 1). Isso pode ter ocorrido devido ao tamanho das lagartas utilizadas no experimento. Com a aplicação do inseticida sobre lagartas grandes, algumas adiantaram seu ciclo, empupando-se alguns dias após a aplicação, e a maioria dos adultos não emergiu. Porém, os adultos que conseguiram emergir comprometeram o cálculo da eficiência no controle do tratamento. Esses resultados são importantes uma vez que, nas aplicações a campo, nem sempre a aplicação pode ser feita quando todas as lagartas são de tamanho pequeno. Com isso, o monitoramento de pragas deve ser constante e eficiente para que o tratamento seja realizado no momento mais adequado.

\section{Avaliação do espectro de gotas}

O bico hidráulico proporcionou gotas de menor tamanho $\left(77,3 \mu \mathrm{m}\right.$ para $\left.\mathrm{DV}_{0,1}\right)$ em relação ao bico rotativo (Tabela 2). Essas gotas, nas condições de campo, são facilmente perdidas pela deriva, não atingindo o alvo e reduzindo a eficiência da aplicação (LEFEBVRE, 1989).

TABELA 2. Diâmetros de gotas nos quais 10\%, 50\% e 90\% do volume total pulverizado apresentaram diâmetros inferiores ao valor do diâmetro indicado, amplitude relativa (A.R.) e percentagem de gotas menores que $100 \mu \mathrm{m}(\% \leq 100)$. Diameter of droplets in which $10 \%, 50 \%$ and $90 \%$ of the total sprayed volume have showed inferior diameters in relation to the indicated diameter, Span (A.R.) and percentage of droplets inferior than $100 \mu \mathrm{m}(\% \leq 100)$.

\begin{tabular}{lccccc}
\hline \multirow{2}{*}{ Variáveis } & \multicolumn{5}{c}{ Diâmetro de Gotas $(\mu \mathrm{m})$} \\
\cline { 2 - 6 } & $\mathrm{DV}_{0,1}$ & $\mathrm{DV}_{0,5}$ & $\mathrm{DV}_{0,9}$ & $\mathrm{AR}$ & $\% \leq 100$ \\
\hline Bico hidráulico & $77,3 \mathrm{~A}$ & $156,4 \mathrm{~A}$ & $281,6 \mathrm{~A}$ & $1,31 \mathrm{~A}$ & $20,0 \mathrm{~A}$ \\
Bico centrífugo & $140,8 \mathrm{~B}$ & $194,1 \mathrm{~B}$ & $283,3 \mathrm{~A}$ & $0,73 \mathrm{~B}$ & $2,3 \mathrm{~B}$ \\
\hline Volume menor & $114,1 \mathrm{~A}$ & $180,7 \mathrm{~A}$ & $279,7 \mathrm{~A}$ & $1,01 \mathrm{~A}$ & $10,6 \mathrm{~A}$ \\
Volume maior & $104,4 \mathrm{~B}$ & $169,8 \mathrm{~B}$ & $275,1 \mathrm{~A}$ & $1,02 \mathrm{~A}$ & $11,6 \mathrm{~A}$ \\
\hline Endosulfan 1,0 L ha & $112,6 \mathrm{~A}$ & $180,2 \mathrm{~A}$ & $286,3 \mathrm{~A}$ & $1,02 \mathrm{~A}$ & $10,9 \mathrm{~A}$ \\
Endosulfan 0,5 L ha & $104,4 \mathrm{~B}$ & $170,3 \mathrm{~B}$ & $271,5 \mathrm{~A}$ & $1,01 \mathrm{~A}$ & $11,3 \mathrm{~A}$ \\
\hline
\end{tabular}

Médias seguidas de mesma letra na coluna não diferem entre si, pelo teste de Tukey $\mathrm{P} \leq 0,05$.

$\mathrm{O}$ diâmetro mediano volumétrico do bico hidráulico $\left(\mathrm{DV}_{0,5}\right)$ foi significativamente menor em relação ao bico rotativo, indicando, ainda, maior risco de deriva; o $\mathrm{DV}_{0,9}$ não diferiu em relação ao tipo de bico. Quanto à uniformidade, verificou-se maior variação no espectro de gotas produzidas pelo bico hidráulico, determinado pelo maior valor de amplitude relativa (Tabela 2). Essa maior uniformidade deve ser a principal responsável pelo desempenho de controle da lagarta, semelhante para os dois bicos avaliados, mesmo com a diferença bastante grande entre os volumes utilizados.

No caso do bico de energia hidráulica, verifica-se que $20 \%$ do volume total aspergido foram de gotas menores que $100 \mu \mathrm{m}$ (Tabela 2), altamente suscetíveis à deriva e à evaporação 
(LEFEBVRE, 1989). Para o bico de energia centrífuga, a percentagem foi de 2,3\%. É importante ressaltar que, na outra ponta do espectro, há gotas que carregam e podem escorrer ou ricochetear da superfície da folha devido ao tamanho e energia excessivos. Mesmo que fiquem sobre o alvo, ainda assim significarão desperdício, por concentrar o produto em áreas restritas à capacidade de espalhamento da gota, em função da calda aspergida. Gotas maiores carregam consigo volume muito maior de calda, pois o dobro do diâmetro significa oito vezes mais volume. Dessa forma, a menor uniformidade do espectro dos bicos hidráulicos compromete parte significativa do volume aspergido, requerendo volume compensatório para a adequada cobertura do alvo, o que complementa a justificativa de que a menor amplitude do espectro do bico hidráulico deve ser a principal responsável pelo bom desempenho em relação ao controle, mesmo com volumes menores. Somente houve diferença de $\mathrm{DV}_{0,1}$ e $\mathrm{DV}_{0,5}$ para volumes de calda e dosagens de inseticidas, porém com efeito relativamente menor em relação aos tipos de bicos (Tabela 2).

CUNHA et al. (2007) relatam que, para as pontas que operam com pressão hidráulica, há formação de gotas bastante desuniformes, dificultando a adequada cobertura do alvo. Com isso, faz-se importante o desenvolvimento de tecnologias que proporcionem a produção de gotas mais uniformes para diminuir a presença de gotas muito pequenas ou de gotas excessivamente grandes.

\section{CONCLUSÕES}

$\mathrm{Na}$ aplicação em laboratório, onde o produto atinge diretamente o alvo, o volume pode ser reduzido até $17 \mathrm{~L} \mathrm{ha}^{-1}$, sem prejudicar o controle de $P$. includens.

A dosagem de $0,5 \mathrm{~L} \mathrm{ha}^{-1}$ do produto comercial endosulfan (recomendada para Anticarsia gemmatalis) não controla satisfatoriamente a lagarta $P$. includens.

O bico rotativo produz gotas de maior uniformidade e menor porcentagem de gotas suscetíveis à deriva.

\section{REFERÊNCIAS}

COSTA, M.A.G.; GRUTZMACHER, A.D.; MARTINS, J.F.S.; COSTA, E.C.; STORCH, G.; STEFANELLLO JÚNIOR, G.J. Eficácia de diferentes inseticidas e de volumes de calda no controle de Spodoptera frugiperda nas culturas do milho e sorgo cultivados em várzea. Ciência Rural, Santa Maria, v.35, n.6, p.1.234-1.242, 2005.

CUNHA, J.P.A.R.; TEIXEIRA, M.M.; FERNANDES, H.C. Avaliação do espectro de gotas de pontas de pulverização hidráulicas utilizando a técnica da difração do raio laser. Engenharia Agrícola, Jaboticabal, v.27, n.1, p.10-15, 2007.

ETHERIDGE, R.E.; WOMAC, A.R.; MUELLER, C.T. Characterization of the spray droplet spectra and patterns of tour venturi-type drift reduction nozzles. Weed Technology,

Champaing, v.13, n.4, p.765-70, 1999.

FERREIRA, M.C. Caracterização da cobertura de pulverização necessária para controle do ácaro Brevipalpus phoenicis (G., 1939) em citros. 2003. 64f. Tese (Doutorado em Produção Vegetal) Faculdade de Ciências Agrárias e Veterinárias, Universidade Estadual Paulista, Jaboticabal, 2003.

FERREIRA, M.C.; MACHADO-NETO, J.G.; MATUO, T. Redução da dose e do volume de calda nas aplicações noturnas de herbicidas em pós-emergência na cultura da soja. Planta Daninha, Viçosa - MG, v.16, n.1, 1998.

HERZOG, D.C. Sampling Soybean Looper on soybean. In: entomology. New York: Springer-Verlag, 1980. p.68-140. . Sampling methods in soybean

KAUR, I.; KUMAR, A.; DURAJA, P. Separation of endosulfan and its metabolites by GC and HPLC. Biomedical Chromatography, Chichester, v.11, n.1, p.33-35, 1997. 
LEFEBVRE, A.H. Atomization and sprays. New York: Hemisphere Publishing Corporation, 1989. $421 \mathrm{p}$.

MATUO, T. Técnicas de aplicação de defensivos agrícolas. Jaboticabal: Funep, 1990. 139 p.

NAKANO, O.; SILVEIRA NETO, S.; ZUCCHI, R.A. Entomologia econômica. São Paulo: USP, 1981. $314 \mathrm{p}$.

OZEKI, Y. Manual de aplicação aérea. São Paulo: Ciba Agro, 2006. 101 p.

PAPA, G.; CELOTO, F.J. Lagartas na soja. 2007. Disponível em:

$<w w w . i l h a s o l t e i r a . c o m . b r / c o l u n a s /$ index.php?acao=verartigo\&idartigo $=1189090532>$. Acesso em: 7 mar. 2008.

PERES, T.; PAPINI, S.; MARCHETTI, M.; LUCHINI, L. Dissipação de endosulfan em amostras de dois tipos de solos brasileiros tratadas em laboratório. Pesticidas: Revista de Ecotoxicologia e Meio Ambiente, Curitiba, v.14, jan./dez. 2004.

REED. J.T.; SMITH, D.B. Droplet size and spray volume effects on insecticide deposit and mortality of heliothine (Lepidoptera: noctuidae) larvae in cotton. Journal of Economic Entomology, v.94, Lanham, n.3, p.640-647, 2001.

SANTOS, J.M.F. Aviação agrícola: manual de tecnologia de aplicação de agroquímicos. São Paulo: Rhodia Agro, 1992. 100 p.

SANTOS, J.M.F.; FEICHTENBERGER, E.; SPÓSITO, M.B.; BELLOTTI, J.A.M. Pulverização. Citricultura Atual, Cordeiropolis, v.10, n.59, 2007.

TEIXEIRA, M.M. Influencia del volumen de caldo y de la uniformidad de distribución transversal sobre la eficacia de la pulverización hidráulica. 1997. 310 f. Tese (Doutorado) - Universidad Politécnica de Madrid, Madrid, 1997. 